\title{
Near-Field Interaction between Domain Walls in Adjacent Permalloy Nanowires
}

\author{
L. O’Brien, D. Petit, H. T. Zeng, E. R. Lewis, J. Sampaio, A. V. Jausovec, D. E. Read, and R. P. Cowburn \\ Nanoscale Magnetics group, Department of Physics, Blackett Laboratory, Imperial College London, \\ Prince Consort Road, London SW7 2AZ, United Kingdom \\ (Received 23 December 2008; published 14 August 2009)
}

\begin{abstract}
The magnetostatic interaction between two oppositely charged transverse domain walls (TDWs) in adjacent Permalloy nanowires is experimentally demonstrated. The dependence of the pinning strength on wire separation is investigated for distances between 13 and $125 \mathrm{~nm}$. The results can be described fully by considering the distribution of magnetic charge within rigid, isolated TDWs. Alternative DW internal structure cannot reproduce this observed dependence. Modeling suggests the TDW internal structure is not appreciably disturbed, and remains rigid although the pinning strength is significant.
\end{abstract}

Quantifying the interaction between magnetic domain walls (DWs) in closely packed networks of ferromagnetic nanowires is of vital importance to recently proposed DW based logic and data storage schemes [1,2], as these interactions could potentially compromise coherent DW propagation and correct device function. In addition to these schemes, DWs in nanowires have also been suggested for use in a wide range of applications, such as atom trapping for quantum information processing [3]. Furthermore, the fundamental properties of DWs themselves are attracting great interest, and DWs can be considered not only as transition regions between oppositely magnetized domains, but as quasiparticles [4]. Their equilibrium [5], dynamic properties [6-9], and interactions with artificial defects [10] are being intensively studied. The ability to hold DWs at defined positions is required for a wide range of spin torque experiments, where current is used to depin the DWs; a continuously variable pinning strength which does not alter the current path is highly desirable in such cases [11].

The effect of the presence of a DW in a nearby structure on the magnetic state of a ferromagnetic ring has been reported [12,13], and quantified in terms of an additional local field due to the DW stray field, but no quantitative analysis of the full pinning potential created by a DW on another DW has been made. In this Letter, we experimentally study the interaction between two oppositely charged transverse DWs (TDWs) traveling in two parallel Permalloy (Py) nanowires. The interwire separations probed in this investigation are below the dimensions of the DW $(\sim 100 \mathrm{~nm})$ itself and so are within the near-field limit. The simulated dependence of the interaction on DW internal structure is examined. By considering the rigid magnetostatic charge distribution of an isolated TDW we are able to reproduce fully the experimental result, suggesting that the internal structure of the DW is not appreciably perturbed by the interaction for the geometries studied. This work opens up the possibility of continuously tailorable, remote DW pinning, which does not affect the DW internal structure. The possibility of a well-defined, tunable pinning potential where the DW acts as a truly rigid quasiparticle is appealing, for example, in current induced resonance experiments $[14,15]$.

Thermally deposited Py nanowires (100 nm wide, $8 \mathrm{~nm}$ thick) were fabricated using electron-beam lithography and a lift-off method. Figure 1(a) shows a scanning electron microscope (SEM) image of a typical structure: two $U$-shaped nanowires with an edge to edge separation, $d$, between 125 and $13 \mathrm{~nm}$, and vertical arm displacement, $W$, equal to $0.5,1$, or $1.5 \mu \mathrm{m}$. In the case shown, $d=100 \mathrm{~nm}$ and $W=1.5 \mu \mathrm{m}$. Magnetic field sequences were applied in the plane of the sample at a frequency of $1 \mathrm{~Hz}$ using a quadrupole electromagnet. Two experiments were performed. In the first experiment, two oppositely charged DWs were created in the nearest pair of corners $\left(A_{1}\right.$ and $A_{2}$ ). In this case [see Fig. 1(b)], the DWs must pass within close proximity of one another before switching the remaining portion of each wire. The attractive interaction between the oppositely charged DWs will therefore directly influence the field required to separate them and switch the rest of the wire. In the second case, DWs are created in corners $B_{1}$ and $B_{2}$ and so switch the central portion of each wire before meeting. This second setup acts as a control experiment to observe switching without any DW-DW interaction.

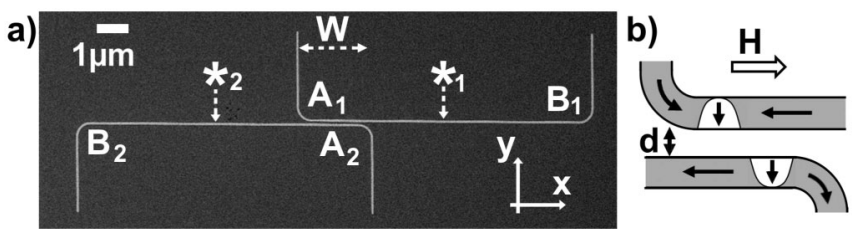

FIG. 1. (a) SEM image of a typical structure showing two $10 \mu \mathrm{m}$ long $U$-shaped Py nanowires separated by an edge to edge distance $d=100 \mathrm{~nm}$. The asterisks indicate the positions where the MOKE laser spot is focused. (b) Schematic of oppositely charged TDWs created in " $A$ " corners moving, under field $H$, along wires separated by $d$. 
For the wire geometries in this investigation $(100 \mathrm{~nm}$ wide, $8 \mathrm{~nm}$ thick) TDWs are stable [16,17]. Within a TDW, the chirality (sense of rotation of magnetization) is determined by the overall charge and magnetization direction of the core of the DW (up or down). To create TDWs at $A(B)$ corners a $45^{\circ}$ field pulse was applied in the $-x,-y$ $(+x,-y)$ direction, giving a downwards head to head (HH) DW at corner $A_{1}\left(B_{1}\right)$ and a downward tail to tail (TT) DW at corner $A_{2}\left(B_{2}\right)$. The field was then decreased to zero at $45^{\circ}$, and a positive (negative) $x$ field was applied to move the two DWs towards one another. The sequence was repeated with all fields reversed to create an upward TT DW at $A_{1}\left(B_{1}\right)$ and an upward HH DW at $A_{2}\left(B_{2}\right)$, moving them towards one another with a negative (positive) $x$ field. Using the field sequences indicated above, the pair of TDWs are initially created with opposite chirality and opposite net charge.

High sensitivity, spatially resolved magneto-optical Kerr effect (MOKE) measurements were used to probe the magnetization of the central portion of each wire [marked with $*$, Fig. 1(a)]. The inset of Fig. 2 is an example of the observed hysteresis loop for the switching of a nanowire. The 70 Oe switching field is due to pinning from mutual interaction between the DWs. The switching fields of portions $*_{1,2}$ of the nanowires were measured as a function of $d$ (measured using SEM images). The results for 90 structures are shown in Fig. 2. Switching of the nanowires in the control experiment (DWs created in corners $B$ )

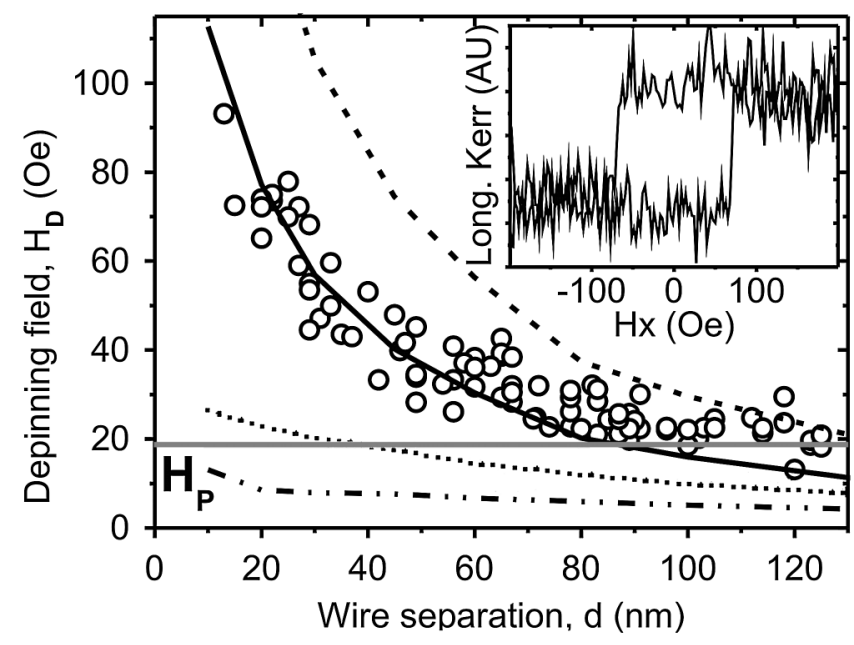

FIG. 2. Open circles: Experimental dependence of DW transmission field $H_{D}$ on wire separation $d$. Gray line, average wire propagation field $H_{P}$. Modeled depinning fields considering charge distribution of the DWs with wide-wide (HHD, TTD; upper long dashed line), wide-narrow (HHU, TTD or HHD TTU; dotted line) or narrow-narrow (HHU, TTU; lower dashed and dotted line) sides closest. Solid black line, wide-wide interaction with a fitting scale factor of 0.54. Inset: Example MOKE observed hysteresis loop of a nanowire. Switching occurs at $H_{D}$, in this case $70 \mathrm{Oe}$, due to depinning from the DW-DW potential well. occurs at an average propagation field $H_{P}=19 \pm 4$ Oe (horizontal gray line). The mean difference between fields $H_{D 1}$ and $H_{D 2}$ at which $*_{1}$ and $*_{2}$ reverse is only 0.1 Oe (standard deviation of $2.1 \mathrm{Oe}$ ) for the case where the DWs interact (DWs created at corners $A$ ); therefore only the average value $H_{D}$ for each structure has been plotted (open circles). We see a monotonic decrease of $H_{D}$ as $d$ increases from a maximum depinning field of 93 Oe measured in wires with $d=13 \mathrm{~nm}$ down to $\sim 19$ Oe $\left(\sim H_{P}\right)$ for $d \sim 90 \mathrm{~nm}$. Above $d \sim 90 \mathrm{~nm}$, the dependence changes as $H_{D}$ remains approximately constant at $H_{P}$. At these high $d$ values the dominant pinning mechanism is due to the inherent roughness of the nanowires and so the interaction between the DWs is no longer measured. No observable dependence was found on $W$. Separate studies, not presented here, ruled out a localized change in wire geometry causing the observed DW pinning [18], suggesting $H_{D}$ is solely due to the interaction between the two DWs. This is also supported by the fact $H_{D 1}$ and $H_{D 2}$ are almost identical for a pair of wires. Furthermore, the narrow distribution of $H_{P}$ (as measured from control experiment) shows we are not measuring a statistical variation of $H_{P}$ along these wires.

To account for the DW-DW interaction dependence on $d$, we describe it in terms of the interaction between magnetic charges, where the magnetic charge density $\rho_{M}$ is defined as $\rho_{M}=-\mu_{0} \boldsymbol{\nabla} \cdot \boldsymbol{M}$ and $\boldsymbol{M}$ is the local magnetization. We expect a model which considers the full DW charge distribution within the wire to match experimentally determined data if the two DWs remain rigid throughout the interaction and we account for finite temperature. OOMMF simulations [19] $\left(3.5 \times 3.5 \times 4 \mathrm{~nm}^{3}\right.$ cell size, $M_{s}=800 \mathrm{kA} / \mathrm{m}, A=13 \mathrm{pJ} / \mathrm{m}$, and damping constant $\alpha=0.5)$ were used to calculate the micromagnetic configuration of the TDW, from which the charge distribution within the DW [20] was obtained. The charge is very inhomogeneously distributed, reflecting the triangular shape of TDWs [16]. In the case of a HH (TT) DW there is a positive (negative) surface charge along the wide edge of the DW, and a smaller negative (positive) charge along the narrow edge [20]. A more complete analysis of the distribution in our wire geometry shows $48 \%$ of the DW charge is located along the wide edge, and has a Gaussian shape. To obtain the strongest interaction achievable between DWs, the TDWs must pass with wide edges closest, which is the case in our setup [see Fig. 1(b)].

The interaction energy $U$ between two extended charge distributions, $D_{1}$ and $D_{2}$, is given by

$$
U=\frac{1}{4 \pi \mu_{0}} \sum_{i \in D_{1}} \sum_{j \in D_{2}} \frac{q_{i} q_{j}}{r_{i j}}
$$

where $r_{i j}$ is the distance between the two charges $q_{i}$ and $q_{j}$. In the case where the two distributions are associated with oppositely charged rigid DWs traveling in two adjacent wires separated by $d$, and $x$ is their lateral separation [see 
Fig. 1(b)], $U(x)$ is a potential well. The change in Zeeman energy $\delta U_{Z}$ caused by separating the DWs by $\delta x$ under a field $H$ is $\delta U_{Z}=-2 M_{s} H S \delta x$, where $M_{s}$ is the saturation magnetization and $S$ is the cross sectional area of the wire. $U(x)$ is sheared by a gradient equal to $-2 M_{s} H S$, and the field required for DW depinning is therefore given by

$$
H_{D}=\left.\frac{1}{2 M_{s} S} \frac{\partial U}{\partial x}\right|_{\max }
$$

The dashed line in Fig. 2 displays $H_{D}^{\mathrm{w}-\mathrm{w}}$ as a function of $d$ when the wide sides of the DWs are closest [HH down (HHD), TT down (TTD)], i.e., the wide-wide interaction. This overestimates the experimental results, but does appear to show the correct trend. This modeling does not include the effect of temperature; however, DW depinning is a thermally assisted process. At room temperature, depinning fields have previously been found to be between $40 \%$ and $70 \%$ of the zero temperature simulated values $[10,20]$. Reference [21] experimentally shows reductions between measurements at 4.2 and $300 \mathrm{~K}$ between $40 \%$ and $60 \%$. The solid black line in Fig. 2 shows a least mean squares fit of the wide-wide model to the experimental data using a scale factor of 0.54 to account for the reduction due to the experiment being conducted at room temperature, a reduction well within previously found values. Fitting is excellent up to $d \simeq 90 \mathrm{~nm}$ above which, as mentioned above, the inherent wire roughness becomes the dominant pinning mechanism.

During propagation, transformations can occur to DW structure via a process known as Walker breakdown [22]; a TDW, of a given charge, oscillates between the two chiralities [up (U) and down (D)] via an intermediate state where an antivortex (AVDW) is nucleated. If breakdown occurs in one or both of our DWs before they meet, the interaction will no longer be with widest sides nearest (w-w; HHD, TTD). Additionally, it has been observed that a TDW may transform into a vortex DW (VDW) (an alternative DW configuration stable in wider or thicker wires) during breakdown [23]. The distances chosen in the experiment over which a DW can propagate $(W<$ $1.5 \mu \mathrm{m})$ before the interaction takes place are below the measured fidelity length to preserve initial chirality [24]. It is not expected, therefore, that breakdown will occur. For completeness, however, in Fig. 2 the modeled depinning fields for the alternative configurations interactions are plotted: wide-narrow (w-n; either HHD, TTU or HHU, TTD by symmetry), $H_{D}^{\mathrm{w}-\mathrm{n}}$, and narrow-narrow (n-n; HHU, TTU), $H_{D}^{\mathrm{n}-\mathrm{n}}$. These interactions have significantly lower depinning fields compared to the experimentally observed interaction, with values $\lesssim H_{P}$, and are clearly not observed for $d \lessgtr 90 \mathrm{~nm}$. We therefore conclude that only the strongest w-w interaction occurs. Figure 3 shows the potential landscapes for the three possible TDW interactions in the case where $d=10 \mathrm{~nm}$. The steep potential well for the w-w interaction accounts for the large observed

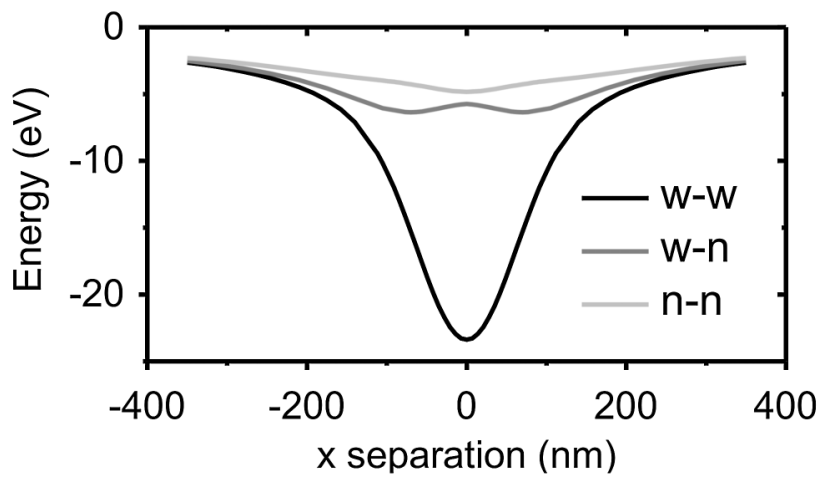

FIG. 3. Potential well obtained when laterally separating a pair of oppositely charged TDWs by $x$ for the case $d=10 \mathrm{~nm}$, from rigid charge model. The nearest sides of the TDWs are widewide (black line), wide-narrow (gray line), and narrow-narrow (light gray line).

$H_{D}$. Note that for the w-n interaction, when the two DWs are closest the interaction is dominated by the repulsion between the two closest line charges, which are of the same sign, giving a local potential barrier. Beyond lateral $x$ separations of $\sim 350 \mathrm{~nm}$ the far-field limit is reached, and all models give the same potential profile as DW internal structure is no longer crucial. To examine the case where the DWs meet during breakdown we consider when a perfect $\mathrm{HH}$ and TT AVDW interact. For the AVDW the only degree of freedom is the core direction. When $d<125 \mathrm{~nm}$, micromagnetic simulation shows that the core of the DWs are expelled as the two DWs meet, restoring the original $\mathrm{w}-\mathrm{w}$ TDW configuration, and is independent of the core directions of the AVDWs.

In addition, micromagnetic simulations were performed, calculating the interaction between oppositely charged TDWs and oppositely charged VDWs. The resulting depinning field dependence on $d$ for TDWs is not shown, but is in good agreement with the near-field charge modeling, providing further evidence that little distortion occurs to the TDW shape. Within a VDW, the internal magnetization rotates around a core, where the magnetization points vertically. This configuration has two internal degrees of freedom, the sense of rotation of the magnetization and core direction. From micromagnetic simulations of all possible HH-TT pairings and $d>80 \mathrm{~nm}$, it is found that the cores are displaced towards one another and all $H_{D}$ are $\lesssim 16$ Oe. For $d \leq 80 \mathrm{~nm}$, i.e., our region of experimental interest, the core is expelled by the interaction, leaving the $\mathrm{w}-\mathrm{w}$ TDW interaction. No significant dependence is found on core direction. The displacement of the core causes clear deformation of the DW and departure of the simulated $H_{D}$ from that obtained using a rigid charge model. This analysis shows we are indeed experimentally measuring the w-w interaction between TDWs, as any other combination would either have a low $H_{D}$ or transform into $\mathrm{w}-\mathrm{w}$ TDWs. The resulting dependence on $d$ is in 
very good agreement with a rigid charge model of TDWs, where the internal structure and chirality of the DW must be considered.

The agreement between modeling and simulation is highly surprising as we only consider magnetostatic charge. This suggests the particular interaction between TDWs of opposite charge leaves the DW structure relatively unperturbed from its free, isolated configuration and that the TDWs can be considered as rigid, isolated quasiparticles. On the contrary, however, VDWs do not appear to act as rigid bodies when they interact. Should a TDW transform into a VDW during propagation, for our region of interest $(d<80 \mathrm{~nm})$, the interaction will cause the DW cores to be expelled and the w-w TDW interaction to result.

Using one DW to pin another is a specific example of pinning by stray fields with little distortion to TDW shape. Reference [25] shows an example of using localized stray fields from multiple magnetic wire ends to create an asymmetric pinning potential, which will act as a DW diode or ratchet similar to the geometrical protrusions in Refs. [26,27]. Using a pinning potential that does not alter wire or TDW shape in spin torque experiments (for example, current induced resonances [15]) may allow clearer interpretation of current based effects. In addition, the interaction between DWs in neighboring nanowires is of great technological importance for DW based logic and data storage devices. Full charge modeling of DWs in nanowires of more technologically relevant dimensions (45 nm wide, $8 \mathrm{~nm}$ thick, $45 \mathrm{~nm}$ spacing) shows DW depinning at $71 \mathrm{Oe}$ at $0 \mathrm{~K}$. This large interaction could result in cross talk errors between adjacent data channels.

In conclusion, we have experimentally studied the interaction between two TDWs of opposite charge moving in parallel magnetic nanowires. The attractive interaction is found to result in depinning fields as high as 93 Oe, measured in wires separated by $13 \mathrm{~nm}$. Modeling using the rigid magnetic charge distributions of isolated DWs reproduces the experimental results extremely well. Comparing results with modeling of DWs in different structural or chiral configurations does not reproduce the experimental results, showing that consideration of the inhomogeneity of the charge is essential to understand near-field DW interactions. Comparing micromagnetic simulations and charge modeling suggests TDWs remain unperturbed by the interaction whereas the VDW core is displaced or ejected, causing departure from a rigid model. Pinning fields at $0 \mathrm{~K}$ of 71 Oe are expected in the technologically relevant dimensions of DW based data storage and logic devices. This study experimentally demonstrates remote pinning using magnetostatic interaction with minimum perturbation to the DWs, opening up the possibility of continuous tailoring of pinning strength with no alteration to the nanowire.

This work was supported by Mr. W. J. Corrigan and the European Community under the Sixth Framework Programme SPINSWITCH (MRTN-CT-2006-035327).

[1] D. A. Allwood et al., Science 309, 1688 (2005).

[2] S. S. P. Parkin, M. Hayashi, and L. Thomas, Science 320, 190 (2008).

[3] D. A. Allwood et al., Appl. Phys. Lett. 89, 014102 (2006).

[4] E. Saitoh et al., Nature (London) 432, 203 (2004).

[5] Y. Nakatani, A. Thiaville, and J. Miltat, J. Magn. Magn. Mater. 290, 750 (2005).

[6] G. S. D. Beach et al., Nature Mater. 4, 741 (2005).

[7] A. Thiaville and Y. Nakatani, Spin Dynamics in Confined Magnetic Structures iii, Topics in Applied Physics Vol. 101 (2006), pp. 161-205.

[8] M. Hayashi et al., Nature Phys. 3, 21 (2007).

[9] J. Yang et al., Phys. Rev. B 77, 014413 (2008).

[10] D. Petit et al., J. Appl. Phys. 103, 114307 (2008).

[11] M. Hayashi et al., Appl. Phys. Lett. 92, 162503 (2008).

[12] M. Klaui et al., Appl. Phys. Lett. 86, 032504 (2005).

[13] M. Laufenberg et al., Appl. Phys. Lett. 88, 212510 (2006).

[14] D. Bedau et al., Phys. Rev. Lett. 99, 146601 (2007).

[15] L. Thomas et al., Nature (London) 443, 197 (2006).

[16] R. D. McMichael and M. J. Donahue, IEEE Trans. Magn. 33, 4167 (1997).

[17] M. Laufenberg et al., Appl. Phys. Lett. 88, 052507 (2006).

[18] Electron-beam lithography proximity effects can cause a local increase in wire width for structures with small $d$. Separate studies of pinning from similar defects give $H_{d}$ values significantly lower than those observed in this experiment.

[19] The OOMMF code is available at http://math.nist.gov/ oommf.

[20] D. Petit et al., Phys. Rev. B 79, 214405 (2009).

[21] A. Himeno et al., J. Magn. Magn. Mater. 286, 167 (2005).

[22] N. L. Schryer and L. R. Walker, J. Appl. Phys. 45, 5406 (1974).

[23] J.-Y. Lee et al., Phys. Rev. B 76, 184408 (2007).

[24] E. R. Lewis et al., Phys. Rev. Lett. 102, 057209 (2009).

[25] See EPAPS Document No. E-PRLTAO-103-064935 for (a) a proposed asymmetric well design using the stray field from magnetostatic charges accumulated at the end of nanowires, (b) the asymmetric well for a TDW due to design (depinning fields are found to be -52 and 168 Oe), and (c) the magnetic surface charge per unit length along the widest edge of a DW for specific geometry discussed. For more information on EPAPS, see http://www.aip.org/ pubservs/epaps.html.

[26] D. A. Allwood et al., Appl. Phys. Lett. 85, 2848 (2004).

[27] A. Himeno et al., J. Appl. Phys. 97, 066101 (2005). 